\title{
Using BiSON to detect solar internal g-modes
}

\author{
J. Kuszlewicz ${ }^{1,2, a}$, G.R. Davies ${ }^{1,2}$, and W.J. Chaplin ${ }^{1,2}$ \\ 1 School of Physics and Astronomy, University of Birmingham, Edgbaston, Birmingham, B15 2TT - \\ United Kingdom \\ 2 Stellar Astrophysics Centre (SAC), Aarhus University, Denmark
}

\begin{abstract}
The unambiguous detection of individual solar internal $g$ modes continues to elude us. With the aid of new additions to calibration procedures, as well as updated methods to combine multi-site time series more effectively, the noise and signal detection threshold levels in the low-frequency domain (where the g modes are expected to be found) have been greatly improved. In the BiSON 23-year dataset these levels now rival those of GOLF, and with much greater frequency resolution available, due to the long time series, there is an opportunity to place more constraints on the upper limits of individual g mode amplitudes. Here we detail recent work dedicated to the challenges of observing low-frequency oscillations using a ground-based network, including the role of the window function as well as the effect of calibration on the low frequency domain.
\end{abstract}

\section{Construction of the BiSON limit spectrum}

The BiSON calibration process now includes a correction for differential extinction, which allows the efficient concatenation of multi-site data by means of a weighted average [1]. As a result, noise levels in the power spectrum have been greatly reduced. However, this improvement in the reduction of the noise levels needs to be coupled with the removal of features caused by the window function in order to be most effective. The complex interaction between the irregular structure in the window function and the data means that getting an accurate model of the contamination caused by the window is crucial.

The best way to account for the contamination caused by the window function is to have a good model for the limit spectrum which can then be convolved with the spectral window, this will aim to take any trends in the window into account (such as the diurnal peaks). The convolved function will then contain all of our knowledge of the noise structure present in the power spectrum, such as granulation and the effect of calibration on the data as well as the contamination caused by the window function.

The construction of the limit spectrum consists of using a four-component Harvey model to take into account the different scales of granulation and active regions, a pink noise component to fit the very low and very high frequency background and a frequency response function which contains all our current knowledge about how the calibration affects the data (using a pragmatic approach as opposed to being physically motivated, for the time being). The model was computed following the method described in Kuszlewicz et al. (in prep.), where $v_{h}$ is the high pass filter cutoff frequency, $v_{c}$ is the Butterworth filter cutoff frequency, $G_{0}$ is the gain at zero frequency and $n$ is the order of the filter.

\footnotetext{
a e-mail: jsk389@bison.ph.bham.ac.uk
}

This is an Open Access article distributed under the terms of the Creative Commons Attribution License 4.0, which permits unrestricted use, distribution, and reproduction in any medium, provided the original work is properly cited. 
EPJ Web of Conferences

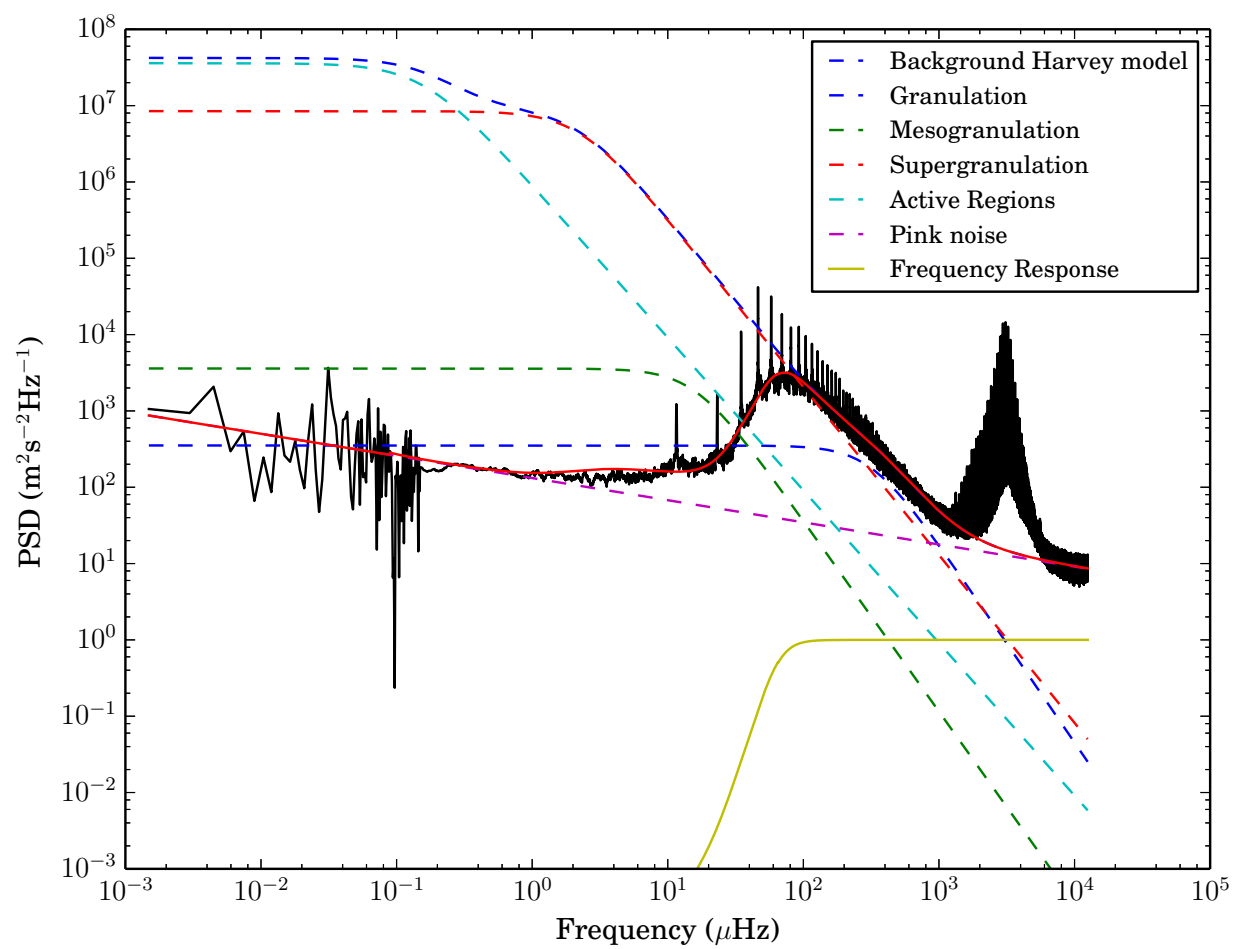

Fig. 1. BiSON limit spectrum constructed using 23 years of BiSON data where the full model is shown in the solid red line and the blue dotted line is the combined background Harvey model. The data has been smoothed using a $0.04 \mu \mathrm{Hz}$ boxcar and the spoilt, smoothed very low frequency region has been replaced with the unsmoothed data.

Table 1. Parameter values for the limit spectrum constructed in figure 1.

\begin{tabular}{llll}
\hline \hline Limit Components & $\tau(\mathrm{s})$ & $\sigma\left(\mathrm{ms}^{-1}\right)$ & $c$ \\
\hline "Granulation" & $5.0 \times 10^{2}$ & 0.42 & 2.6 \\
"Mesogranulation" & $1.0 \times 10^{4}$ & 0.3 & 2.5 \\
"Supergranulation" & $7.0 \times 10^{4}$ & 5.5 & 2.2 \\
"Active Regions" & $1.0 \times 10^{6}$ & 3.0 & 2.0 \\
\hline \hline Noise Components & $a$ & $\alpha$ & - \\
\hline Pink Noise & 2.4 & 0.29 & - \\
\hline \hline Frequency Response & $\mathrm{G}_{0}$ & $v_{c}(\mu \mathrm{Hz})$ & $n$ \\
\hline Butterworth & 1.0 & $6.5 \times 10^{1}$ & 3.0 \\
\hline \hline Frequency Response & $\mathrm{G}_{0}$ & $v_{h}(\mu \mathrm{Hz})$ & $n$ \\
\hline High-pass & - & $5.5 \times 10^{2}$ & - \\
\hline \hline
\end{tabular}

\section{References}

1. Davies, G.R., Chaplin, W.J., Elsworth, Y., and Hale, S.J., MNRAS 441, (2014) 3009-3017 